University of Nebraska - Lincoln

DigitalCommons@University of Nebraska - Lincoln

1999

\title{
Routing Algorithms for All-Optical Networks with Power Considerations: The Unicast Case
}

\author{
Maher Ali \\ University of Nebraska-Lincoln \\ Byrav Ramamurthy \\ University of Nebraska-Lincoln, bramamurthy2@unl.edu \\ Jitender S. Deogun \\ University of Nebraska-Lincoln, jdeogun1@unl.edu
}

Follow this and additional works at: https://digitalcommons.unl.edu/cseconfwork

Part of the Computer Sciences Commons

Ali, Maher; Ramamurthy, Byrav; and Deogun, Jitender S., "Routing Algorithms for All-Optical Networks with Power Considerations: The Unicast Case" (1999). CSE Conference and Workshop Papers. 67.

https://digitalcommons.unl.edu/cseconfwork/67

This Article is brought to you for free and open access by the Computer Science and Engineering, Department of at DigitalCommons@University of Nebraska - Lincoln. It has been accepted for inclusion in CSE Conference and Workshop Papers by an authorized administrator of DigitalCommons@University of Nebraska - Lincoln. 


\title{
Routing Algorithms for All-Optical Networks with Power Considerations: The Unicast Case
}

\author{
Maher Ali, Byrav Ramamurthy ${ }^{1}$, Jitender S. Deogun \\ Department of Computer Science \& Engg. \\ University of Nebraska-Lincoln \\ Lincoln, NE 68588-0115, U.S.A. \\ Email:\{mali, byrav, deogun\}@cse.unl.edu
}

\begin{abstract}
In this paper, we investigate the problem of routing connections in all-optical networks while allowing for degradation of routed signals by different optical components. To overcome the complexity of the problem, we divide it into two parts. First, we solve the pure RWA problem using fixed routes for every connection. Second, power assignment is accomplished by either using the smallest-gain first (SGF) heuristic or using a genetic algorithm. Numerical examples on a wide variety of networks show that (a) the number of connections established without considering the signal attenuation was most of the time greater than that achievable considering attenuation and (b) the genetic solution quality was much better than that of SGF, especially when the conflict graph of the connections generated by the linear solver is denser.
\end{abstract}

\section{INTRODUCTION}

Wavelength division multiplexing (WDM) is an important technique employed for taking advantage of the enormous bandwidth in fiber optics [1]. An optical network consists of an interconnection of stations, switches and other devices using optical fibers. A connection in an optical network is set up on a lightpath. A lightpath between two stations is an available wavelength on a series of fiber links from source to destination such that the route is simple (i.e., free of cycles) and the signal remains in the optical domain (i.e., it is not converted to electronics). An important problem in WDM networks is the routing and wavelength assignment (RWA) problem [2]. The RWA problem involves selecting the best combination of route (path) and wavelength for each connection in a given demand matrix such that the number of connections established is maximized and no two connections which share a common link use the same wavelength. Stations are interconnected together using cross-connect switches (XCS's) (or simply switches). An XCS can have zero or more local stations connected to it. These stations are connected using an array of receivers and transmitters (lasers or LEDs). The stations together with the XCS are referred to as a wavelength-routed node (WRN) [3]. A station is assumed to be able to transmit/receive on any available wavelength. Figure 1 shows a sample wavelength-routed all-optical network based on the ARPA-2 topology.

\footnotetext{
${ }^{1}$ This work was supported in part by a UNL Research Council Grant-In-Aid award.
}

\section{A. Motivation and Related Work}

Power issues in optical networks were first addressed in $[4,5,6]$. The setting was a broadcast-and-select network, where the number of wavelengths at every point of the network is known beforehand. The problem investigated was to place optical amplifiers on the links such that all signals can be carried. To the best of our knowledge, the physical aspects of a network and its effect on routing and wavelength assignment solution were first investigated by Sabella et al. [7]. In [7], the investigators first solved the problem without considering the physical aspects of the network. Each route generated is then analyzed from the transmission point of view. Three different routing schemes (WP, VWP, and PVWP) were compared using the same traffic pattern. The average number of connections established that assure acceptable transmission performance was used as the main metric for comparisons. The transmission performance of individual connections was measured by the accumulation of the amplified spontaneous emission (ASE) along the links and the in-band crosstalk inside the switch. The inline amplifiers were assumed to exactly compensate for the loss of power in the fiber segments, and no attempt is made to optimize the signal power in the network. Another recent study [3] investigates the impact of transmission impairments in an optical network for a dynamic setting. However, in most previous studies (e.g., $[2,8]$ ), the optical network is modeled under the perfect conditions assumption and only the logical aspect of the problem is considered. A connection between two stations which traverses optical fiber links and encounters different devices is assumed to maintain valid power levels on the entire path from source to destination. In reality, optical signals do degrade. Amplification, whether inside the switching devices or on fiber links, is needed to compensate for these losses. A proper power level for the individual signal in the optical network must be maintained such that the aggregate power (found by summing up the power levels of all signals) on a fiber does not exceed a certain value. What makes things even more difficult is that a realistic EDFA amplifier model is nonlinear. This means that the amplifier cannot work at full capacity all the time, and does get saturated. Saturation occurs when the total input power of the amplifier exceeds a specific threshold. When this situation occurs, the gain available for individual signals is no longer the small signal gain (i.e., a reduction in gain occurs).

In this paper, we refer to the problem of routing and wavelength assignment in WDM networks with power consideration as RWA-P. The following gives a formal definition of the RWA-P problem. 
Definition Routing and Wavelength Assignment with Power ( $R W A-P$ ) Problem. Given a network topology represented by a graph $G=(V, E, \Delta, W)$, where $V$ is the set of nodes, $E$ is the set of links, $\Delta(l)$ is the set of amplifier locations preplaced on link $l$, and $W$ is the set of wavelengths available on a link. Also given is a traffic matrix, $T_{|V| \times|V|}$, representing the number of lightpaths requested from any station to any other station, and system parameter triple $<p_{\text {sen }}, P_{\text {max }}, S S G>$, where $p_{\text {sen }}$ is the threshold for a signal below which a signal cannot be detected; $P_{\max }$ is the maximum aggregate power on a link, and $S S G$ is the maximum small-signal gain for an EDFA amplifier. The objective is to maximize the traffic in the network by routing connections, assigning wavelengths, and determining the levels of transmission powers for connections while ensuring that a connection is routed using the same wavelength on all links, no two connections which share a link use the same wavelength, the signal is maintained above the threshold (i.e., $\geq p_{s e n}$ ) from the source to the destination, and the aggregate power value of all the signals on a link should be $\leq P_{\max }$.

This paper is organized as follows. First, the device models and network architecture are discussed in Section II. Next, the two-phase solution approach is presented in Section III. Section IV gives numerical examples for different network topologies and various traffic demands. Finally, Section V concludes this paper and highlights some future work.

\section{PhysiCAL Limits of DeVICES}

In this section, we present some of the physical aspects of the RWA problem. One proposed solution for the RWA-P problem is also outlined.

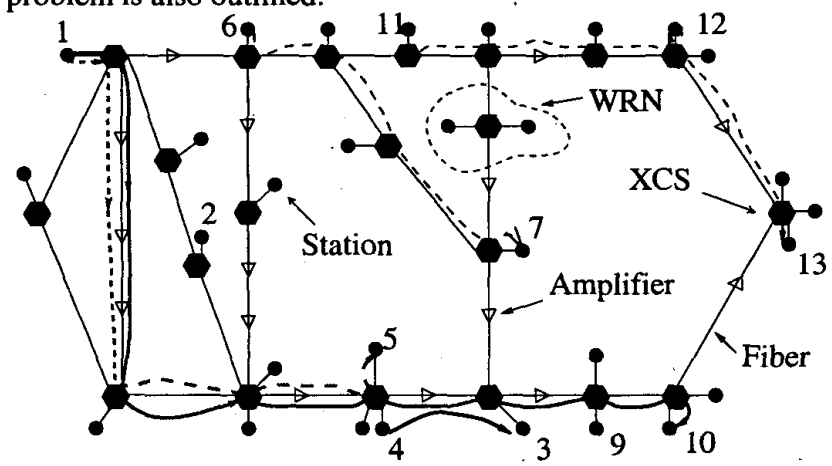

Figure 1: A sample wavelength-routed all-optical network based on the ARPA-2 topology. Six lightpaths are established.

\section{A. Network and Node Architecture}

A pair of unidirectional fibers connects the wavelength routing nodes (WRNs) in the network. Long fiber links may be interspersed with inline optical amplifiers (e.g., EDFA). Traffic on a WDM channel can be transferred from one link to another at a WRN. A wavelength routing node contains components such as taps, input/output amplifiers, multiplexers, etc [3]. The WRN also contains an optical cross-connect (XCS). This XCS utilizes multiplexers/demultiplexers and wavelength routing switches (WRS) to realize all different traffic permutations. A signal entering a WRN encounters various components which contribute to power loss/gain. An optical tap is needed in the WRN to tap into the power of an input or output fiber link. In addition, demultiplexers/multiplexers degrade the optical signal while splitting/joining the individual channels on a fiber link. The switching power loss for WRS number $i$ is given by $L O S S_{i}=2\left\lceil\log _{2}\left(D_{i}\right)\right\rceil L_{s}+4 L_{w}$, where $D_{i}$ is the node degree (i.e., number of fiber links and stations attached to this WRN), $L_{s}$ is the insertion loss, and $L_{w}$ is the coupling loss [9]. In order to establish a connection in this network, we not only need a free wavelength on a route, but also need enough power on the signal for it to reach the receiving station.

\section{B. Amplifier Gain Model}

The gain available at an amplifier is given by the following function

$$
G\left(P_{i n}, S S G\right)=\min \left\{S S G,\left(P_{\max }-P_{i n}\right)\right\}
$$

where $P_{i n}$ is the total input power, $P_{\max }$ is the maximum amplifier output power, and $S S G$ is the small signal gain. During its operation, the amplifier is either in the linear or the saturation region. If it is in the linear region, the gain available is the full small-signal gain. When the total input power is increased above a specific threshold, however, the amplifier can no longer provide the full small-signal gain and the amplifier enters the saturation region. We assume equal gain for all connections (on different wavelengths) entering a specific amplifier regardless of their individual power levels. In the literature, this is referred to as the flat gain assumption. Also, in this paper we assume the same fixed pump power (i.e., same small-signal gain) for all inline amplifiers. The value used in this study for the small-signal gain is $20 \mathrm{~dB}$.

\section{Physical Characteristics of Devices}

Two system parameters constrain the signals in the network. The sensitivity level, $p_{s e n}$, is the minimum power required on an individual wavelength such that the signal can be detected. Also, the aggregate power of all wavelengths on a fiber cannot exceed a certain value, $P_{\max }$. Every device in the network either amplifies or degrades the signal. For example, an optical fiber degrades the signal at a rate of $0.2 \mathrm{~dB} / \mathrm{km}$. The values of the other device parameters (e.g., tap loss, switch insertion loss, multiplexer loss, etc.) are typical and can be found in [10].

\section{Added Complexity}

Since we require the signal to maintain proper power levels in the network, additional constraints are added to the pure RWA formulation so that the power at the beginning and at the end of each optical component is checked. For more information on the RWA-P problem formulation please refer to $[10]$.

\section{TWO-PHASE SOLUTION APPROACH}

Since the formulation of the RWA-P problem turned out to be a mixed-integer nonlinear [10], we divide the problem into 
two separate sub-problems. First, a linear program is solved for the pure RWA problem (routing and wavelength assignment without power considerations). We use CPLEX MILP solver [11] to solve the linear program. Then given this fixed number of connections, one can try to establish connections by assigning appropriate transmission powers at the source stations. Figure 2 shows the overall solution methodology.

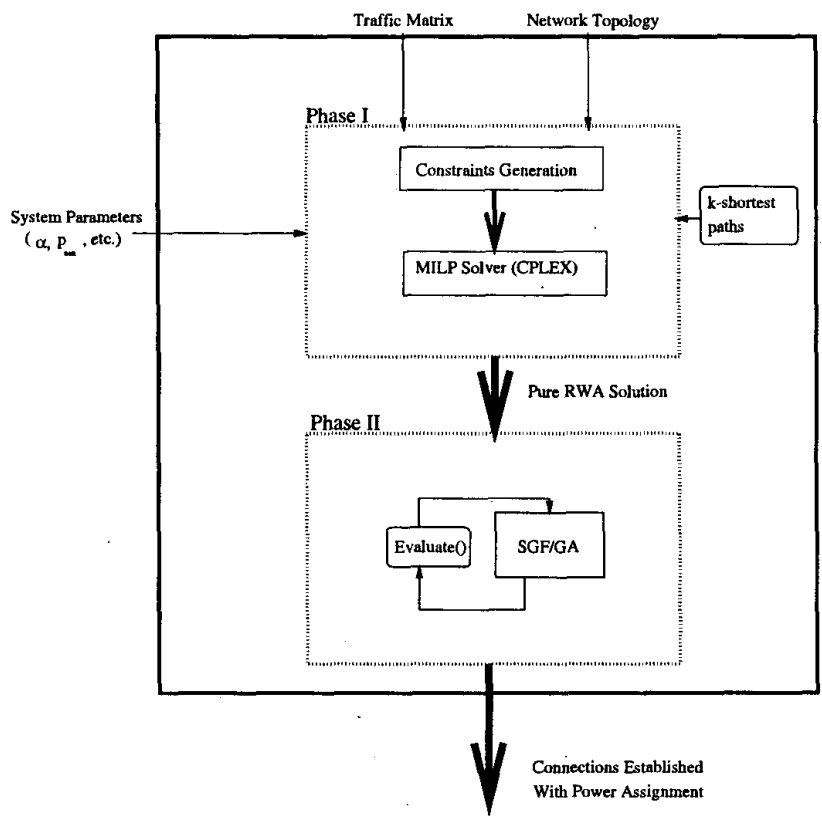

Figure 2: Two-Phase Solution Approach.

For the linear program, we chose to use for each station pair, a set of fixed routes for satisfying connection requests, an approach more general than using only the shortest-path. However, since even using one path for each connection is an NP-hard problem, only the shortest-path is used. We present the smallest-gain first heuristic (SGF) in Subsection A and the genetic algorithm $(G A)$ in Subsection B.

\section{A. Smallest Gain First (SGF)}

As shown in Fig. 2, the power assignment problem is handled by either a genetic algorithm or by a heuristic that we call the smallest-gain first (SGF) heuristic. Given the $m$ connections obtained by solving the ILP, we sort the connections according to Eqn. (2). Eqn. (2) finds the total amount of gain/loss that connection $i$ encounters along its path assuming that no other connections are present in the network. By assuming that the amplifiers are uniformally distributed on the links, the SGF heuristic establishes connections starting with those which require minimum network resources (i.e., will have less effect on future connections).

$$
g_{i}=\sum_{l \in \text { path }[i]}\left(\operatorname{LOSS}_{d_{l}}+\sum_{c \in l} \Upsilon_{l, c}(0)\right)
$$

In the formula above, path $[i]$ denotes the set of ordered links for connection $i ; \Upsilon_{l, c}(0)$ denotes the loss/gain of component

\author{
Function Evaluate $(\mathcal{C})$ \\ ${ }^{*}$ Input: $\mathcal{C}$ set of connections */ \\ /* Output: Number of established connections */ \\ begin \\ $/^{*} \#$ of established connections is initially zero */ \\ Number $\leftarrow 0$ \\ $k \leftarrow|\mathcal{C}|$ \\ $\gamma^{*}$ for all connections in $\mathcal{C}$ *I \\ for $i=1, i<=k$ \\ if AddConnection $\left(\mathcal{C}_{i}\right)$ \\ Number $\leftarrow$ Number +1 \\ end if \\ end for \\ $f^{\star}$ return the number of established connections */ \\ return $N u m b e r$ \\ end Function \\ Figure 3: Function Evaluate(). Given an ordered list of connections, it \\ assigns powers one by one in the order given.
}

$c$ on link $l$ assuming that no other connections enter the same component; $d_{l}$ is the node which is the destination of link $l$; and $L O S S_{h}$ is the switching loss of switch $h$. The function Evaluate() adds the connections one by one in the given order. If connection $k$ is being added and it causes some connection say $j(j<k)$ to drop below sensitivity level (due to loss of gain), connection $k$ is rejected and the next connection, $k+1$, is tried and so on.

\section{B. Genetic Algorithm}

Genetic Algorithms (GA) are stochastic search optimization methods that are widely used in combinatorial optimization and parameter tuning applications. GA tries to emulate a phenomenon observed in nature: survival of the fittest. GA applies this to global optimization problems. An individual in GA is basically a solution for the problem we are trying to optimize. A solution consists of a set of variables. Each variable can have a value taken from a domain. In our problem setting, the solution is a set of $m$ real numbers drawn from $\left[0, P_{\max }\right]$. The value of the variable, $t_{i}$, corresponds to the transmission power of the source station of connection $i$. A collection of solutions is referred to as a population. Since GA tries to mimic nature, at any discrete time, $t$, the current population is referred to as the $t^{t h}$ generation. By moving from one generation to another over time, the quality of the population is improved. To move from generation $t$ to generation $(t+1)$, we use three genetic operators that have been widely researched and used. The first operator is the crossover which exploits the solutions in the current population. Two mutation operators were used: creep and RandomStore to diversify and explore other regions of the search space [12].

The genetic algorithm we employ in this study is referred to as steady-state genetic algorithm. In every iteration, two individuals are selected to reproduce the children. The children are then mutated with some probability and evaluated using the 
Evaluate() function in Fig. 3. Finally, the children are inserted in the population relative to their score and the process repeats. The process is terminated if the number of iterations exceeds a specific value or the population converges. It is worth noting that employing an iterative approach like GA allows us to trade time with solution quality. The population size used was 20 . The rate of crossover, creep, and RandomStore was set to $60 \%$, $25 \%$, and $5 \%$, respectively.

\section{NUMERICAL EXAMPLES}

The linear programs were solved using CPLEX [11] on a lightly-loaded Sun Ultra-60 workstation. The two heuristics (see Fig. 2) were run on lightly-loaded $200 \mathrm{MHz}$ Pentium II PC running Windows NT 4.0 and using Microsoft foundation classes (MFC). Consider the 6-node mesh optical network given in [10]. Table 1 shows the results obtained by varying the number of wavelengths, for the demand matrix, $T$, where $T_{i, j}$ is the number of connections desired from station $i$ to station $j$, given in (3).

$$
T=\left[\begin{array}{llllll}
0 & 3 & 2 & 0 & 2 & 1 \\
0 & 0 & 3 & 0 & 0 & 0 \\
0 & 1 & 0 & 1 & 0 & 3 \\
1 & 3 & 2 & 0 & 3 & 2 \\
0 & 0 & 0 & 3 & 0 & 0 \\
1 & 2 & 3 & 1 & 2 & 0
\end{array}\right]
$$

For each case in Table 1 , the first column shows the number of available wavelengths on a link. The second column gives the number of connections generated by the linear solver (which serves as an upper bound on the number of connections for the heuristics) and the time (in seconds) it took to solve the linear program. In the third column, the number of established connections using the SGF heuristic is given and the time it took in seconds followed by the percentage of established connection relative to the LP solution. The last two columns give the results for the genetic algorithm solution. We find that the genetic algorithm-based heuristic performs better than the SGF heuristic. For small $\mathrm{W}$, the results obtained from our GA-based heuristic achieves the upper bound on the number of connections. When the number of wavelengths is increased, more connections can be routed using the linear solver. The increased number of connections from the LP solution however, forces an increase in the GA solution time and a decrease in the $(G A / L P)$ ratio. The time difference between the SGF and GA is justified since the SGF heuristic evaluates only one point of the search space, while the GA evaluates two points in every generation (assuming we use the steady-state method).

Table 2 shows the results obtained when all other variables are kept fixed except the traffic matrix. Four different traffic patterns were used: (a) $U[1, W]:$ A random value from $[1, W]$ is stored in the $T_{i j}$ 's entry with probability 0.3 , (b) $\frac{W}{2}$ : A constant value $\left(\frac{W}{2}\right)$ is stored in each traffic matrix entry, (c) $W$ : A maximum number of lightpaths $(W)$ is stored in each entry and (d) $U[0,1]:$ A value of 1 is stored in entry $T_{i j}$ with probability 0.3 , and 0 otherwise. We find that all four cases

\begin{tabular}{||l|l|l|l|l|l||}
\hline W & LP & SGF & $\frac{S G F}{L P}$ & GA & $\frac{G A}{L P}$ \\
\hline 6 & 28 & 24 & $85 \%$ & 28 & $100 \%$ \\
& $0.74 \mathrm{~s}$ & $0.01 \mathrm{~s}$ & & $151.10 \mathrm{~s}$ & \\
\hline 7 & 31 & 26 & $83 \%$ & $31 \mathrm{~s}$ & $100 \%$ \\
& $0.93 \mathrm{~s}$ & $0.01 \mathrm{~s}$ & & $160.30 \mathrm{~s}$ & \\
\hline 8 & 33 & 27 & $81 \%$ & 31 & $93 \%$ \\
& $1.52 \mathrm{~s}$ & $0.01 \mathrm{~s}$ & & $197.37 \mathrm{~s}$ & \\
\hline 9 & 35 & 28 & $80 \%$ & 33 & $94 \%$ \\
& $1.82 \mathrm{~s}$ & $0.01 \mathrm{~s}$ & & $254.12 \mathrm{~s}$ & \\
\hline 10 & 37 & 27 & $72 \%$ & 33 & $89 \%$ \\
& $2.01 \mathrm{~s}$ & $0.01 \mathrm{~s}$ & & $330.02 \mathrm{~s}$ & \\
\hline 11 & 38 & 27 & $71 \%$ & 34 & $89 \%$ \\
& $2.23 \mathrm{~s}$ & $0.01 \mathrm{~s}$ & & $394.30 \mathrm{~s}$ & \\
\hline
\end{tabular}

Table 1

Traffic matrix in (3) applied to the mesh network in [10] using the shortest path while varying the number of wavelengths and using the shortest path. (Time measured in seconds.)

show a justification for spending additional time by using the genetic algorithm. In case (d), however, GA's improvement over SGF is small because there is not much interaction between connections due to the small number of connections output from the linear solver.

\begin{tabular}{||l|l|l|l|l|l||}
\hline Demand & LP & SGF & $\frac{S G F}{L P}$ & GA & $\frac{G A}{L P}$ \\
\hline $\mathrm{U}[1, W]$ & 33 & 27 & $81 \%$ & 31 & $93 \%$ \\
& $1.52 \mathrm{~s}$ & $0.01 \mathrm{~s}$ & & $197.37 \mathrm{~s}$ & \\
\hline$\frac{W}{2}$ & 48 & 35 & $72 \%$ & 44 & $91 \%$ \\
& $4.31 \mathrm{~s}$ & $0.01 \mathrm{~s}$ & & $189.02 \mathrm{~s}$ & \\
\hline$W$ & 48 & 35 & $72 \%$ & 44 & $91 \%$ \\
& $0.67 \mathrm{~s}$ & $0.01 \mathrm{~s}$ & & $167.00 \mathrm{~s}$ & \\
\hline $\mathrm{U}[0,1]$ & 15 & 13 & $86 \%$ & 14 & $93 \%$ \\
& $2.03 \mathrm{~s}$ & $0.01 \mathrm{~s}$ & & $115.00 \mathrm{~s}$ & \\
\hline
\end{tabular}

Table 2

Using different traffic demands for the mesh network in [10] with $W=8$ and using the shortest path

Table 3 shows the computational results obtained on different proposed physical topologies for all-optical networks. The high-speed Italian network (Fig. 4 from [7]) with 21 nodes and 114 unidirectional fiber links is taken as a representative of future realistic wavelength-routed all-optical networks. Amplifiers were placed on the links every $100 \mathrm{~km}$. The Pacific Bell network [3] is a 15 station mesh network with 72 unidirectional links. It was chosen as a representative of medium-sized interconnection of rings network. A Hypercube network with 16 stations and 96 unidirectional links is also used in the study. In addition, a double-rooted binary tree with 14 stations and 54 unidirectional links is used. A 12-node bidirectional ring was also used. Finally, a $3 \times 3$ grid network with 9 stations and 42 unidirectional links is used. The demand matrices (with $0-1$ entries) used were generated randomly (by storing a 1 in $T_{i j}$ 's entry with probability 0.3 ). Table 3 shows that as the traffic increases, spending more time using the GA 
is justifiable. In the case of the bidirectional ring and the Italian network, the clique size of the routes conflict graph was large. ${ }^{2}$ This forces a large interaction between the connections which in turn increases the search space. It is clear that this increase in the search space reduces the chances of finding near optimal solutions (in a reasonable time) by the genetic algorithm (about $50 \%$ of the LP). In the case of the hypercube on the other hand, there is not much interaction between connections (i.e., connections are localized because of the inherent structure of the hypercube) which results in a large number of connections being established by the genetic algorithm.

\begin{tabular}{|c|c|c|c|c|c|}
\hline Topology & LP & SGF & $\frac{S G F}{L P}$ & $\overline{\mathrm{GA}}$ & $\frac{G A}{L P}$ \\
\hline $\begin{array}{l}\text { Italian [7] } \\
N=21 \\
L=114\end{array}$ & $\begin{array}{l}53 \\
35.92 \mathrm{~s}\end{array}$ & $\begin{array}{l}22 \\
0.07 \mathrm{~s}\end{array}$ & $41 \%$ & $\begin{array}{l}26 \\
485.40 \mathrm{~s}\end{array}$ & $49 \%$ \\
\hline $\begin{array}{l}\text { Pacific Bell } \\
N=15 \\
L=72\end{array}$ & $\begin{array}{l}14 \\
0.63 \mathrm{~s}\end{array}$ & $\begin{array}{l}12 \\
0.03 \mathrm{~s}\end{array}$ & $85 \%$ & $\begin{array}{l}13 \\
275.60 \mathrm{~s}\end{array}$ & $92 \%$ \\
\hline $\begin{array}{l}\text { Hypercube } \\
N=16 \\
L=96\end{array}$ & $\begin{array}{l}33 \\
0.78 \mathrm{~s}\end{array}$ & $\begin{array}{l}20 \\
0.14 \mathrm{~s}\end{array}$ & $60 \%$ & $\begin{array}{l}26 \\
338.6 \mathrm{~s}\end{array}$ & $78 \%$ \\
\hline $\begin{array}{l}\text { Binary Tree } \\
N=14 \\
L=54\end{array}$ & $\begin{array}{l}21 \\
0.66 \mathrm{~s}\end{array}$ & $\begin{array}{l}10 \\
0.02 \mathrm{~s}\end{array}$ & $47 \%$ & $\begin{array}{l}11 \\
284.00 \mathrm{~s}\end{array}$ & $52 \%$ \\
\hline $\begin{array}{l}\text { Bi. Ring } \\
N=12 \\
L=48\end{array}$ & $\begin{array}{l}17 \\
0.45 \mathrm{~s}\end{array}$ & $\begin{array}{l}9 \\
0.02 \mathrm{~s}\end{array}$ & $52 \%$ & $\begin{array}{l}9 \\
482.14 \mathrm{~s}\end{array}$ & $52 \%$ \\
\hline $\begin{array}{l}\text { Grid } \\
N=9 \\
L=42\end{array}$ & $\begin{array}{l}9 \\
0.25 \mathrm{~s}\end{array}$ & $\begin{array}{l}7 \\
0.01 \mathrm{~s}\end{array}$ & $77 \%$ & $\begin{array}{l}7 \\
240.30 \mathrm{~s}\end{array}$ & $77 \%$ \\
\hline
\end{tabular}

Table 3

Experimental results on different network topologies, where $W=8$ and using the shortest path. Traffic demand was randomly generated. $N$ is the number of local stations, and $L$ is the number of unidirectional links.

\section{CONCLUSION AND FUTURE WORK}

In this paper, we introduced the problem of routing and wavelength assignment in optical networks while considering power constraints. To overcome the complexity due to nonlinear constraints, we devised a two-phase hybrid solution approach employing genetic algorithms (GA) and Smallest-Gain First heuristic (SGF). We compared GA-based approach to the SGF solution approach. The results indicate that the GA-based approach performs well for a wide choice of parameters. The additional time spent searching using the genetic algorithm always results in a better solution (larger number of established connections).

Connection management and signal monitoring are integral parts of any network management infrastructure developed

\footnotetext{
${ }^{2}$ The conflict graph for a set of connections in a network is found by creating a node for every connection. An edge is added between two nodes iff these connections share a link.
}

for large-scale WDM optical networks. We plan to study the performance of our algorithms for a wide range of network architectures such as those capable of multicasting. We also plan to investigate the related problem of network design Given a network, a demand matrix, and a library of device models, how to optimally place components such as amplifiers, so as to maximize the number of connections established?

\section{REFERENCES}

[1] B. Mukherjee, Optical Communication Networks. New York : McGraw-Hill, 1997.

[2] R. Ramaswami and K. N. Sivarajan, "Routing and wavelength assignment in all-optical networks," IEEE/ACM Transactions on Networking, vol. 3, pp. 489500 , Oct 1995.

[3] B. Ramamurthy, D. Datta, H. Feng, J. P. Heritage, and B. Mukherjee, "Impact of transmission impairments on the teletraffic performance of wavelength-routed optical networks," IEEE/OSA Journal of Lightwave Technology, Oct. 1999 (to appear).

[4] C.-S. Li, F. F.-K. Tong, C. J. Georgiou, and M. Chen, "Gain equalization in metropolitan and wide area optical networks using optical amplifiers," in Proceedings, IEEE INFOCOM '94, (Toronto, Canada), pp. 130-137, June 1994.

[5] B. Ramamurthy, J. Iness, and B. Mukherjee, "Optimizing amplifier placements in a multi-wavelength optical LAN/MAN: The equally powered-wavelengths case," IEEE/OSA Journal of Lightwave Technology, vol. 16, pp. 1560-1569, Sept. 1998.

[6] B. Ramamurthy, J. Iness, and B. Mukherjee, "Optimizing amplifier placements in a multi-wavelength optical LAN/MAN: the unequally powered-wavelengths case," IEEE/ACM Transactions on Networking, vol. 6, pp. 755767, December 1998.

[7] R. Sabella, E. Iannone, M. Listanti, M. Berdusco, and S. Binetti, "Impact of transmission performance on path routing in all-optical transport networks," IEEE/OSA Journal of Lightwave Technology, vol. 16, pp. 1965-1972, Nov. 1998.

[8] B. Mukherjee, D. Banerjee, S. Ramamurthy, and A. Mukherjee, "Some principles for designing a widearea WDM optical network," IEEE/ACM Transactions on Networking, vol. 4, pp. 684-696, Oct 1996.

[9] R. A. Spanke, "Architectures for guided-wave optical space switching systems," IEEE Communications Magazine, vol. 25, May 1987.

[10] M. Ali, B. Ramamurthy, and J. S. Deogun, "Routing and wavelength assignment (RWA) with power considerations in optical networks," in Proceedings, IEEE GLOBECOM '99, (Rio De Janeiro, Brazil), December 5-9 1999 (to appear).

[11] http://www.cplex.com.

[12] D. Lawrence, Handbook of genetic algorithms. New York: Van Nostrand Reinhold, 1991. 